\title{
FRET Reveals the Formation and Exchange Dynamics of Protein- Containing Complex Coacervate Core Micelles
}

\author{
Antsje Nolles, ${ }^{\dagger, \dagger}$ Ellard Hooiveld, ${ }^{\dagger}$ Adrie H. Westphal, ${ }^{\dagger,}{ }^{\S}$ Willem J. H. van Berkel, ${ }^{\dagger} \odot$ J. Mieke Kleijn, ${ }^{\dagger}$ \\ and Jan Willem Borst ${ }^{*}, \dagger, \S_{0}$
}

${ }^{\dagger}$ Laboratory of Biochemistry, ${ }^{\ddagger}$ Physical Chemistry and Soft Matter, and ${ }^{\S}$ MicroSpectroscopy Centre Wageningen, Wageningen University \& Research, Stippeneng 4, 6708 WE Wageningen, The Netherlands

Supporting Information

ABSTRACT: The encapsulation of proteins into complex coacervate core micelles (C3Ms) is of potential interest for a wide range of applications. To address the stability and dynamic properties of these polyelectrolyte complexes, combinations of cyan, yellow, and blue fluorescent proteins were encapsulated with cationic-neutral diblock copolymer poly(2-methyl-vinyl-pyridi-

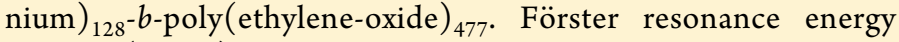
transfer (FRET) allowed us to determine the kinetics of C3M formation and of protein exchange between C3Ms. Both processes

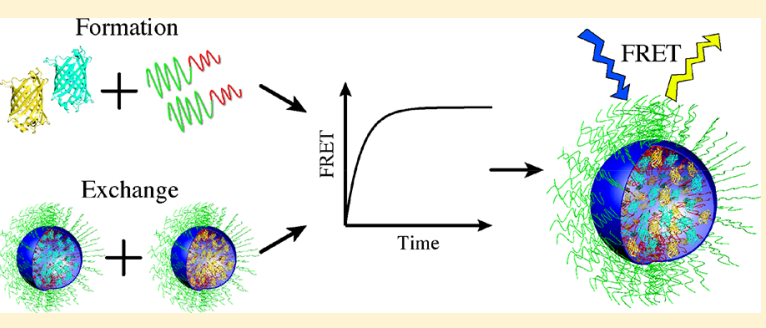
follow first-order kinetics with relaxation times of $\pm 100 \mathrm{~s}$ at low ionic strength $(I=2.5 \mathrm{mM})$. Stability studies revealed that $50 \%$ of FRET was lost at $I=20 \mathrm{mM}$, pointing to the disintegration of the C3Ms. On the basis of experimental and theoretical considerations, we propose that C3Ms relax to their final state by association and dissociation of near-neutral soluble proteinpolymer complexes. To obtain protein-containing C3Ms suitable for applications, it is necessary to improve the rigidity and salt stability of these complexes.

\section{INTRODUCTION}

The encapsulation of proteins is important in many applications, such as the controlled delivery of functional ingredients in foods, medical formulations, industrial enzymatic processes, and protein storage. In all cases, adequate encapsulation is required while keeping the bioactivity or functionality intact. Micelles composed of polyelectrolyte complexes, so-called complex coacervate core micelles $(\mathrm{C} 3 \mathrm{Ms})$, are promising structures for protein protection, stabilization, and controlled delivery. ${ }^{1-6} \mathrm{C} 3 \mathrm{Ms}$ are simple to prepare by mixing protein solutions with oppositely charged diblock copolymer solutions in stoichiometric charge ratios. ${ }^{7,8}$ Micellar structures are then spontaneously formed and are small enough to remain in solution. The inner core of $\mathrm{C} 3 \mathrm{Ms}$ has a high loading capacity; hundreds of protein molecules can be incorporated into one micelle. ${ }^{1}$ The micellar core provides a relatively water-rich environment, thereby shielding protein molecules from the bulk solution while protein structure and functionality are preserved and any immune response is reduced. Because the formation of $\mathrm{C} 3 \mathrm{Ms}$ is driven by electrostatic interactions, contributed by Coulombic attraction and entropic counterion release, ${ }^{9-11}$ the $\mathrm{pH}$ and ionic strength strongly influence the formation, dynamics, and stability of these structures.

While the structure and morphology of C3Ms have been the subject of many studies, the stability and dynamic aspects of micelles and the exchange of molecules between individual C3Ms have been scarcely investigated. In 1998, Cohen Stuart and co-workers were the first to study the rate of formation of
C3Ms, composed of poly((dimethylamino)ethyl-methacrylate)-co-poly(glyceryl-methacrylate) (PDMAEMA-coPGMA) and poly(acrylic-acid $)_{158}\left(\mathrm{PAA}_{158}\right)$, using dynamic light scattering. ${ }^{7}$ They found that micelle formation took place on time scales of 0.01 to $100 \mathrm{~s}$ depending on the ionic strength. Before equilibrium was reached, a relatively high transient turbidity was found, which they attributed to macroscopic phase separation (the formation of transient large aggregates) on short time scales $(<1 \mathrm{~ms})$, after which the dense phase rearranges into C3Ms. A corresponding molecular model was tested using self-consistent field theory. ${ }^{12}$ Similar results were found by Hofs et al. ${ }^{13}$ for C3Ms consisting of poly([4-(2aminoethylthio)butylene $]$ hydrochloride $)_{49}-b$-poly(ethyleneoxide $)_{212}\left(\mathrm{PAETB}_{49}-b-\mathrm{PEO}_{212}\right)$ and poly(acrylic-acid) (PAA) of various lengths. For the formation of $\mathrm{C} 3 \mathrm{Ms}$ from poly ((methacryloyloxyethyl)trimethylammonium chloride $)_{530}$ $\left(\right.$ PMOTAC $\left._{530}\right)$ and poly(ethylene oxide) $)_{113}$ - $b$-poly(methacrylic acid $)_{122}$ or $294\left(\mathrm{PEO}_{113}-b\right.$ - $\mathrm{PMAA}_{122}$ or 294$)$ at an ionic strength of $10 \mathrm{mM}$, Holappa et al. ${ }^{14}$ found two processes occurring on different time scales: a process with a kinetic coefficient of $0.0126 \mathrm{~s}^{-1}$, which was attributed to the "insertion and expulsion" of single chains, and a slow process with a kinetic coefficient of $7.77 \times 10^{-5} \mathrm{~s}^{-1}$, which was attributed to the "merging and splitting" of C3Ms. The final equilibrium state of C3Ms was reached only after about $16 \mathrm{~h}$. The authors

Received: April 17, 2018

Revised: September 10, 2018

Published: September 13, 2018 
discussed their findings in terms of a model that was introduced by Dormidontova ${ }^{15}$ describing the micellization of polymeric surfactants (hydrophilic-hydrophobic block copolymers). Zhang et al. ${ }^{16}$ also referred to this model for the formation of their C3Ms in deionized water composed of poly(ethylene-oxide) $)_{113}$ - $b$-poly(sodium-4-styrene-sulfonate $)_{47}$ $\left(\mathrm{PEO}_{113}-b\right.$-PSSNa 47$)$ and poly(ethylene-oxide $)_{113}-b$-poly(quaternized-2-(dimethylamino) ethyl-methacrylate) ${ }_{48}$

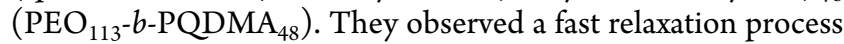
(within $2.6 \mathrm{~ms}$ ), which they related to initial quasi-equilibrium complex formation. A slower process (within $0.4 \mathrm{~s}$ ) was attributed to structural rearrangements leading to final equilibrium complexes and was supposed to proceed through a micelle fusion-fission mechanism. Very long relaxation times were reported by Lindhoud et al. ${ }^{4}$ for two different proteincontaining three-component $\mathrm{C} 3 \mathrm{Ms}$, i.e., $\mathrm{PAA}_{42}-b-\mathrm{PAAm}_{417} /$ PDMAEMA $_{150} /$ lysozyme (poly(acrylic-acid) $_{42}$ - $b$-poly(acylamide $)_{417}$ and poly $(N, N \text {-dimethylaminoethyl-methacrylate) })_{150}$, respectively) and $\mathrm{P}_{2} \mathrm{MVP}_{41}-b-\mathrm{PEO}_{205} / \mathrm{PAA}_{139} / \alpha$-lactalbumin (poly(2-methyl-vinyl-pyridinium-iodide) ${ }_{41}$ - $b$-poly(ethyleneoxide $)_{205}$ and poly(acrylic-acid) ${ }_{139}$, respectively), at low ionic strength. For lysozyme-containing C3Ms, a relaxation time of 2 days was found, while C3Ms containing $\alpha$-lactalbumin did not reach equilibrium in their experiment of 2 weeks. Differences in relaxation behavior were explained from the diversity in molecular properties, in particular, the nature of used polyelectrolytes: weak or strong. The authors pointed to the possible advantage of quenched systems in which over longer periods no rearrangements in micelles occur so that proteins are really trapped and cannot leave them.

Most of the studies mentioned above concern the dynamics of C3Ms formed by the coacervation of two polyelectrolyte chains. Only the systems of Lindhoud et al. involved proteincontaining C3Ms, which is also the focus of this article. ${ }^{17}$ Understanding the dynamics and stability of proteincontaining $\mathrm{C} 3 \mathrm{Ms}$ under different conditions is crucial for designing functional protein-containing micellar structures. In the present study, Förster resonance energy transfer (FRET) between mTurquoise2 (a cyan fluorescent protein) and SYFP2 (strongly enhanced yellow fluorescent protein 2) was used to study the kinetics of formation of C3Ms, the dynamics of protein exchange between these structures, and their salt stability. An important difference with the protein-containing structures described by Lindhoud et al. is that no homopolymer with the same charge sign as the protein is incorporated in $\mathrm{C} 3 \mathrm{Ms} .{ }^{17}$ As a result, many more protein molecules can be accommodated in one micelle (several hundred instead of about 10), while the micellar structures are much more dynamic.

Previously, we have compared the degree of encapsulation of a range of Aequorea victoria $(A V)$ - and Anthozoa-derived fluorescent proteins (FPs) and the effect on their spectral properties. ${ }^{17}$ The encapsulation of these FPs was established with the use of cationic-neutral diblock copolymer poly(2methyl-vinyl-pyridinium $)_{128}$ - $b$-poly (ethylene-oxide) 477 $\left(\mathrm{P}_{2} \mathrm{MVP}_{128}-b-\mathrm{PEO}_{477}\right)$, which is also used in the present study. Furthermore, for most of the FPs, including the three $A V$ derived FPs used in the present study, encapsulation was performed at $\mathrm{pH}$ 9, at which they have a negative charge of 911. On the basis of similar preferred micellar compositions (PMCs $(\sim 0.7))$, C3M sizes $(\sim 34.5 \mathrm{~nm})$, and encapsulation efficiencies $(\sim 100 \%)$, we concluded that all $A V$-derived FPs are encapsulated in the same way and with the same affinity.
The results further showed that mTurquoise 2 and SYFP2 are the best candidates for FRET studies regarding the dynamics of C3Ms. For both mTurquoise2 and SYFP2, encapsulation in $\mathrm{C} 3 \mathrm{Ms}$ resulted in only minor changes in fluorescence properties. Next to that, mTurquoise 2 has a high quantum yield $\left(\Phi_{\text {mTurquoise2 }}=0.93\right)$ and SYFP2 has a high extinction coefficient $\left(\varepsilon_{\text {SYFP2,515nm }}=101000 \mathrm{M}^{-1} \mathrm{~cm}^{-1}\right) .{ }^{18,19}$ Therefore, we have selected these two FPs for the present study, while SBFP2 (strongly enhanced blue fluorescent protein 2) was used as an invisible protein substitute to lower the fluorescence signal of interest while keeping the total protein concentration constant. ${ }^{17}$

On the basis of the results of the presented kinetic FRET experiments in this study, we propose a model to describe the formation and relaxation of protein-containing C3Ms. To roughly quantify the Gibbs energy of $\mathrm{C} 3 \mathrm{M}$ formation using this model, we take the number of proteins encapsulated in one micelle as 450 and the critical micelle concentration (CMC) at the PMC as $100 \mathrm{nM}$ protein, as previously found for enhanced green fluorescent protein (EGFP), ${ }^{1}$ which is an $A V$ derived FP as well. The obtained insights also allow us to develop strategies for generating a tunable stability of micelles for different applications.

\section{EXPERIMENTAL SECTION}

Materials. Poly(2-vinylpyridine) ${ }_{128}$ - $b$-poly(ethylene-oxide $)_{477}$ $\left(\mathrm{P}_{2} \mathrm{VP}_{128}-b-\mathrm{PEO}_{477}\right.$, Polymer Source Inc., Canada, $M_{\mathrm{w}} / M_{\mathrm{n}}=1.10$, $M_{n}=34.5 \mathrm{~kg} / \mathrm{mol}$ ) was quaternized following a procedure described elsewhere. ${ }^{1}$ For $\mathrm{P}_{2} \mathrm{MVP}_{128}-b-\mathrm{PEO}_{477}\left(M_{n}=50.8 \mathrm{~kg} / \mathrm{mol}\right)$, a final degree of quaternization of approximately $87 \%$ was obtained, implying that the polymer bears approximately 112 positive charges. A stock solution of $\mathrm{P}_{2} \mathrm{MVP}_{128}-b-\mathrm{PEO}_{477}(50 \mu \mathrm{M})$ was prepared by dissolving the polymer in $10 \mathrm{mM}$ borate buffer $(\mathrm{pH} 9.0)$, and the solution was stored at $-20{ }^{\circ} \mathrm{C}$. All solutions were filtered through $0.20 \mu \mathrm{m}$ poly(ether sulfone) membrane syringe filters (Advanced Microdevices Pvt. Ltd., Ambala Cantt, India). All other chemicals were from commercial sources and of the purest grade available.

Protein Production. The genes of mTurquoise2 and SYFP2-His were cloned into bacterial expression vector pTYB12 (New England Biolabs Inc., Ipswich, MA, USA) to generate FP fusions with a chitinbinding domain and an intein. ${ }^{20-22}$ The cDNA of SBFP2 in a pRSETb vector was kindly provided by Dr. Joachim Goedhart, University of Amsterdam. ${ }^{23}$ For protein production, E. coli BL21 cells were used. Details on protein production and purification are described elsewhere. ${ }^{2}$ After on-column cleavage, mTurquoise 2 and SYFP2-His were acquired without the chitin-binding domain. SBFP2 and SYFP2 still contained the His tag after purification. Purified protein stock solutions of mTurquoise2 $(21.7 \mu \mathrm{M})$, SYFP2 $(29.5$ $\mu \mathrm{M})$, and SBFP2 $(35.5 \mu \mathrm{M})$ were stored in $10 \mathrm{mM}$ borate buffer $(\mathrm{pH}$ 9.0) at $4{ }^{\circ} \mathrm{C}$.

Protein concentrations were determined with a Pierce BCA protein assay (Pierce Biotechnology, Rockford, IL, USA) using a bovine serum albumin standard as a reference. The purity of all fluorescent proteins was checked by SDS-PAGE.

Absorption and Steady-State Fluorescence Spectroscopy. Absorption spectra of $1 \mu \mathrm{M}$ SBFP2, mTurquoise2, or SYFP2 in 10 $\mathrm{mM}$ borate buffer ( $\mathrm{pH}$ 9.0) were recorded on a Hewlett-Packard 8453 diode array spectrophotometer at $20^{\circ} \mathrm{C}$. Spectrophotometer settings were controlled using the UV-visible ChemStation software package (Hewlett-Packard, Palo Alto, CA, USA).

Steady-state fluorescence spectra were recorded on a Cary Eclipse spectrofluorimeter (Varian Inc., Middelburg, The Netherlands). Excitation and emission slits were set to yield bandwidths of $5 \mathrm{~nm}$. Samples were measured in $1 \mathrm{~mL}$ quartz cuvettes with a path length of $1 \mathrm{~cm}$. Fluorescence emission spectra of $1 \mu \mathrm{M}$ mTurquoise 2 and SYFP2 in $10 \mathrm{mM}$ borate buffer ( $\mathrm{pH} 9.0$ ) were separately measured at excitation wavelengths of 440 and $490 \mathrm{~nm}$, respectively. FRET was 
determined by using fluorescence intensities from donor $(475 \mathrm{~nm})$ and acceptor channels (527 nm, Figure 1). For the FRET

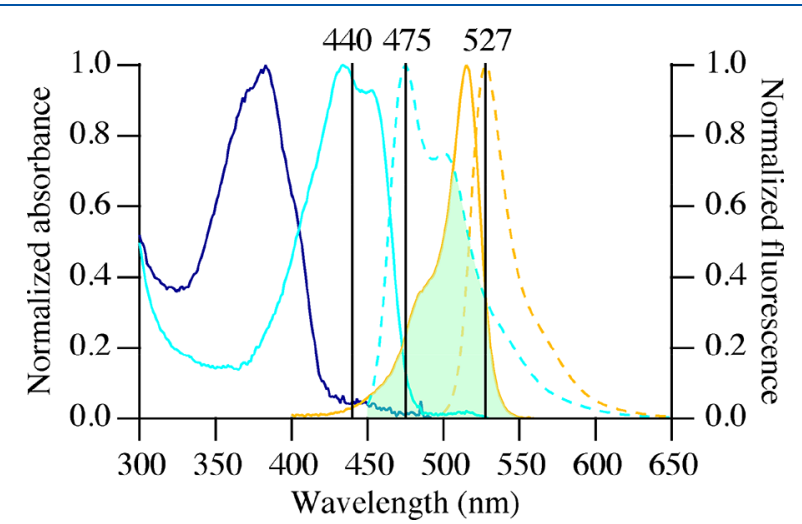

Figure 1. Normalized absorption spectra of SBFP2 (blue line), mTurquoise2 (cyan solid line), and SYFP2 (orange solid line) and fluorescence emission spectra of mTurquoise2 (cyan dashed line) and SYFP2 (orange dashed line). The listed wavelengths at the top of the graph indicate the following from left to right: excitation wavelength of mTurquoise2 (donor excitation), emission wavelength of mTurquoise2 (donor channel), and emission wavelength of SYFP2 (acceptor channel). The green area indicates the spectral overlap between mTurquoise2 and SYFP2.

experiments, fluorescence emission spectra were obtained using an excitation wavelength of $440 \mathrm{~nm}$, and emission was recorded between 450 and $600 \mathrm{~nm}$. All measurements and incubations prior to the measurements were performed at $20^{\circ} \mathrm{C}$.

C3M Preparations. In general, the encapsulation of FPs with polymers into $\mathrm{C} 3 \mathrm{Ms}$ was achieved by first diluting fluorescent protein stock solutions in $10 \mathrm{mM}$ borate buffer ( $\mathrm{pH} 9.0)$ to a final protein concentration of $1 \mu \mathrm{M}$ and a FRET pair composition of $1: 1$ (mTurquoise2/SYFP2), followed by the addition of polymer to a final concentration of $0.18 \mu \mathrm{M}$. C3Ms were prepared at a $F^{+}$ratio of 0.70 , which is the $\mathrm{PMC}$ at $\mathrm{pH} 9.0$ of proteins with diblock copolymer $\mathrm{P}^{2} \mathrm{MVP}_{128}-b-\mathrm{PEO}_{477 .}{ }^{17}$ The PMC is expressed as $F^{+}=\left[n_{+}\right] /\left(\left[n_{+}\right]+\right.$ $\left.\left[n_{-}\right]\right)$, with $\left[n_{+}\right]=c_{+} N_{+}$referring to the total concentration of positively charged groups on the polymer and $\left[n_{-}\right]=c_{-} N_{-}$being the total concentration of negatively charged groups on the protein molecules.

The net charges of FPs as a function of $\mathrm{pH}$ were calculated using the PROPKA 3.1 software package ${ }^{24,25}$ At $\mathrm{pH} 9.0$, the charges $\left(N_{-}\right)$ of SBFP2, mTurqouise2, and SYFP2 are $-8.96,-11.30$, and -9.75 , respectively, which are used to calculate $\left[n_{-}\right]$.

Equilibrium States of C3Ms with Differing FRET Pair Amounts. Protein solutions with different amounts of the FRET pair were obtained by varying the FRET pair/SBFP2 ratio between 0.1 and 1.0. C3Ms were formed by mixing the protein solution with an aliquot of a stock solution of $\mathrm{P}_{2} \mathrm{MVP}_{128}-b-\mathrm{PEO}_{477}$. The resulting solutions were left to equilibrate for $3 \mathrm{~h}$ prior to recording the fluorescence emission spectra.

Formation of C3Ms. A protein solution of a 50\% FRET pair was prepared (25\% mTurquoise2, 25\% SYFP2, and 50\% SBFP2) to follow the formation of $\mathrm{C} 3 \mathrm{Ms}$ at the PMC. An equilibrated protein solution and an aliquot of a stock solution of $\mathrm{P}_{2} \mathrm{MVP}_{128}-b-\mathrm{PEO}_{477}$ were mixed rapidly in a quartz cuvette and placed in a fluorescence spectrophotometer sample compartment. Fluorescence emission spectra were recorded with a time interval of $30 \mathrm{~s}$. The obtained data set was fitted using IGOR Pro 6.11 (Wavemetrics, Inc., Lake Oswego, OR, USA).

Protein Exchange between C3Ms. Two separate C3M solutions were prepared, one with mTurquoise2-C3Ms and one with SYFP2-C3Ms but both with varying amounts of SBFP2, so that after mixing the final FRET pair/SBFP2 ratios varied between 0.20 and 0.50 . The $\mathrm{C} 3 \mathrm{M}$ solutions were left to equilibrate for $3 \mathrm{~h}$ prior to the measurement. To follow the protein exchange between $\mathrm{C} 3 \mathrm{Ms}$, equilibrated solutions of mTurquoise2-C3Ms and SYFP2-C3Ms with the same SBFP2 amounts were mixed rapidly in a quartz cuvette and placed in the fluorescence spectrophotometer sample compartment. Fluorescence emission spectra were recorded with a time interval of $30 \mathrm{~s}$. The obtained data were fitted using global analysis with IGOR Pro 6.11 .

Salt Titration of C3Ms. To test the salt stability of proteincontaining C3Ms, first a solution of C3Ms containing a 50\% FRET pair was prepared at the PMC. Next, by the addition of small aliquots of a $500 \mathrm{mM} \mathrm{NaCl}$ solution, the $\mathrm{NaCl}$ concentration was gradually changed from 0 (initial) to 37.0 (final) $\mathrm{mM}$, corresponding to an ionic strength change from 2.5 to $39.5 \mathrm{mM}$. To determine the dilution effect of the addition of $\mathrm{NaCl}$, a control experiment was performed by adding corresponding volumes of $10 \mathrm{mM}$ borate buffer $(\mathrm{pH} 9.0)$ to preformed 50\% FRET pair C3Ms. Fluorescence emission spectra were recorded at the start of the experiment and after every addition of $\mathrm{NaCl}$ or buffer.

Dynamic Light Scattering. Dynamic light scattering (DLS) measurements were performed on an ALV instrument equipped with a $22 \mathrm{~mW}$ Uniphase model $1145 \mathrm{P}$ HeNe laser operating at $632.8 \mathrm{~nm}$ and an ALV/Dual High QE ADP detector unit with a fiber splitting device for two detectors connected to an external ALV7004 multiple tau digital correlator (ALV-Laser Vertriebsgesellschaft m-b.H., Langen, Germany). The detection angle, $\theta$, was set at $90^{\circ}$, and all measurements were performed at $20^{\circ} \mathrm{C}$.

For the determination of the salt stability of C3Ms, $500 \mu \mathrm{L}$ solutions of $50 \%$ FRET pair C3Ms were prepared at the PMC. $\mathrm{NaCl}$ concentrations ranging from 0 (initial) to $37.0 \mathrm{mM}$ (final) were obtained upon addition from a stock solution, corresponding to an ionic strength change from 2.5 to $39.5 \mathrm{mM}$.

DLS autocorrelation curves were generated from 10 individual intensity traces and averaged. The CONTIN method ${ }^{26,27}$ was used to analyze the distribution of the radii of the C3Ms. The data were analyzed with the AfterALV data analysis software program (AfterALV 1.0d, Dullware, The Netherlands).

FRET Data Analysis. FRET is a spectroscopic phenomenon that is applied in different research disciplines to study (bio)molecular interactions. ${ }^{28,29}$ This phenomenon involves nonradiative energy transfer from a fluorescent donor to an acceptor and can take place only if the following three prerequisites are met: (1) there is an overlap between donor fluorescence emission and acceptor excitation spectra, (2) the distance between both fluorophores is in the range of $1-10 \mathrm{~nm}$, and (3) there is an appropriate orientation of the dipole moments of both fluorophores. These three conditions determine the propensity of energy transfer, which is reported as FRET efficiency. Most fluorescent proteins emit in the visible spectrum and have overlapping spectra and therefore are widely used for FRET experiments. $^{30,31}$ The most effective combination for FRET is the pair formed by cyan (CFP) and yellow (YFP) fluorescent proteins.

C3Ms containing mTurquoise 2 and SYFP2 display FRET, which is demonstrated in Figure 2 (red solid line), showing that the fluorescence emission of SYFP2 takes place upon excitation of mTurquoise2 (excitation at $440 \mathrm{~nm}$, Figure 1). Furthermore, steadystate fluorescence data in Figure 2 shows that in FRET experiments the cross-talk of mTurquoise2, i.e., the fluorescence emission of the donor at $527 \mathrm{~nm}$, and direct excitation of SYFP2 at $440 \mathrm{~nm}$ occur. Both contributions were taken into account for the calculation of the overall FRET. The ratio of the SYFP2 to mTurquoise2 fluorescence intensities $\left(R_{\mathrm{FRET}}\right)$ is expressed as

$$
R_{\mathrm{FRET}}=f_{D_{\mathrm{ex}}}^{A_{\mathrm{em}}} / f_{D_{\mathrm{ex}}}^{D_{\mathrm{em}}}
$$

with $f_{D_{\mathrm{ex}}}^{A_{\mathrm{ex}}}$ being the measured fluorescence intensity in the acceptor channel upon donor excitation, which is corrected for spectral crosstalk and direct excitation of the acceptor, and $f_{D_{\mathrm{ex}}}^{D_{\mathrm{em}}}$ being the measured fluorescence intensity in the donor channel after donor excitation, which is corrected for detection efficiencies of the dyes following a procedure described elsewhere. ${ }^{32}$ Both corrected parameters are also used to calculate the transfer efficiency, $E$ : 


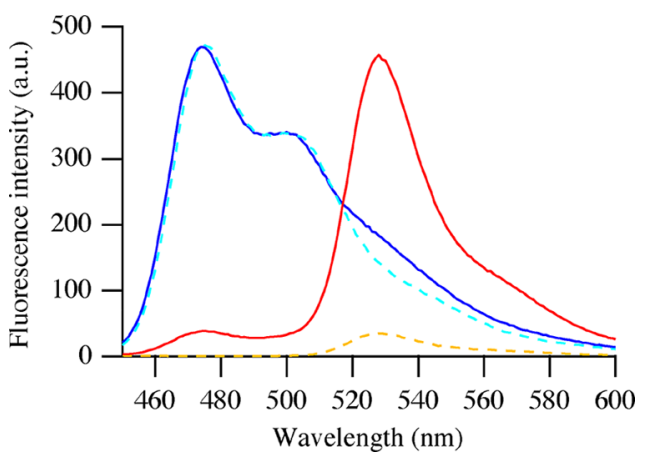

Figure 2. Steady-state fluorescence emission spectra of mixed C3Ms containing mTurquoise2 and SYFP2 (red solid line), of mixed mTurquoise2 and SYFP2 free in solution (blue solid line), of mTurquoise2 in C3Ms (cyan dashed line), and of SYFP2 in C3Ms (orange dashed line). Proteins are at equimolar concentrations with a total concentration of $1 \mu \mathrm{M}$, and excitation was at $440 \mathrm{~nm}$.

$$
E=f_{D_{\mathrm{ex}}}^{A_{\mathrm{em}}} /\left(f_{D_{\mathrm{ex}}}^{D_{\mathrm{em}}}+f_{D_{\mathrm{ex}}}^{A_{\mathrm{em}}}\right)
$$

The Förster radius is calculated using

$$
R_{0}=0.211\left(\kappa^{2} n^{-4} \Phi_{\mathrm{D}} J(\lambda)\right)^{1 / 6}
$$

and

$$
J(\lambda)=\int_{0}^{\infty} f_{\mathrm{D}}(\lambda) \varepsilon_{\mathrm{A}}(\lambda) \lambda^{4} \mathrm{~d} \lambda
$$

where $\Phi_{\mathrm{D}}$ is the donor quantum yield in the absence of an acceptor $\left(\Phi_{\text {mTurquoise2 }}=0.93\right), n$ is the refractive index of the intervening solution $(n=1.41),{ }^{2}$ which was calculated from the change in fluorescence lifetimes of EGFP and mEGFP molecules inside the core of the micelles compared to that of the proteins free in solution, and $\kappa^{2}$ is the orientation factor describing the relative orientation of the transition dipoles of donor and acceptor $\left(\kappa^{2}=0.475\right) .^{33,34}$ The overlap integral, $J$, is a function of the wavelength, $\lambda$, and is calculated using $f_{\mathrm{D}}(\lambda)$ and $\varepsilon_{\mathrm{A}}(\lambda)$ as the wavelength-dependent emission spectrum of the donor and the wavelength-dependent molecular extinction coefficient of the acceptor $\left(\varepsilon_{\mathrm{SYFP} 2,515 \mathrm{~nm}}=101000 \mathrm{M}^{-1}\right.$ $\mathrm{cm}^{-1}$ ), respectively.

For the calculation of the Förster radius, $R_{0}$, between mTurquoise 2 and SYFP2 in the micelles, we used some results from our previous work. ${ }^{17}$ The emission spectrum of encapsulated mTurquoise 2 and the excitation spectrum of encapsulated SYFP2 were used in eq 4 . In eq 3, a refractive index of 1.41 for the micellar core was used, which was determined from the decrease in fluorescence lifetimes upon encapsulation of EGFP and mEGFP. ${ }^{2}$ Furthermore, a $\kappa^{2}$ value of 0.475 was used because the fluorescent proteins are restricted in their movement inside the C3Ms. ${ }^{33,34}$ On the basis of these values, a Förster radius of $53.4 \AA$ was calculated for mTurquoise2 and SYFP2 inside the C3Ms. Herewith, the second prerequisite of FRET is met. Furthermore, a Förster radius of $53.4 \AA$ is larger than the dimensions of an FP, which are $24 \AA$ in diameter and $42 \AA$ in height. ${ }^{35,36}$

Gibbs Energy of Association of C3Ms as a Function of Ionic Strength. The protein-containing C3Ms display salt-dependent association/dissociation behavior, which follows an apparent twostate equilibrium process (Supporting Information). Therefore, the equilibrium can be described as

$$
\mathrm{C} 3 \mathrm{M}_{\text {dissociated }} \rightleftharpoons \mathrm{C} 3 \mathrm{M}_{\text {associated }}
$$

with its corresponding equilibrium constant

$$
K_{\text {association }}=\left[\mathrm{C} 3 \mathrm{M}_{\text {associated }}\right] /\left[\mathrm{C} 3 \mathrm{M}_{\text {dissociated }}\right]
$$

The shift in the equilibrium toward the dissociation of the C3Ms upon increasing the ionic strength is due to the screening of the electrostatic interactions between the negatively charged proteins and the positively charged diblock copolymers. At sufficiently high salt concentrations, FRET will not take place because the micelles have fallen apart and the distance between the FPs is large, resulting in high donor and low acceptor fluorescence intensities. At low salt concentration, the C3Ms are stable and thus the FPs are in close proximity, which leads to a high FRET efficiency.

The increase in the donor fluorescence intensity and the corresponding decrease in the acceptor fluorescence intensity display sigmoidal behavior as a function of ionic strength. Figure S4 shows the ionic strength dependence of these fluorescence intensities, normalized between their minimum and maximum values. We calculated $K_{\text {association }}$ at each ionic strength from the normalized fluorescence intensities of the donor and acceptor and its increase and decrease, respectively, compared to the start of the salt titration (0 $\mathrm{mM} \mathrm{NaCl}$ ). These normalized fluorescence intensities are proportional to the concentration of $\mathrm{C} 3 \mathrm{Ms}$ and the concentration of dissociated C3Ms, respectively.

The Gibbs energy of association $\left(\Delta G_{\text {association }}^{0}\right)$ of $\mathrm{C} 3 \mathrm{Ms}$ was calculated at each salt concentration by applying eq 7

$$
\Delta G_{\text {association }}^{0}=-R T \ln \left(K_{\text {association }}\right)
$$

with gas constant $R$ and absolute temperature $T$. A plot of $\Delta G_{\text {association }}^{0}$ against ionic strength, $I$, yields a straight line according to

$$
\Delta G_{\text {association }}^{0}=\Delta G_{\text {association,water }}^{0}+m I
$$

where $\Delta G_{\text {association,water }}^{0}$ is the Gibbs energy of C3M formation at zero ionic strength and $m$ is the slope of the line.

\section{RESULTS}

FRET Efficiency in C3Ms with Varying FRET Pair Concentrations. Figure 2 shows the fluorescence emission spectra of equimolar amounts of mTurquoise 2 and SYFP2 free in solution as well as encapsulated in C3Ms upon excitation at $440 \mathrm{~nm}$. Encapsulating mTurquoise2 and SYFP2 together in $\mathrm{C} 3 \mathrm{Ms}$ yields a strong reduction of the donor fluorescence intensity and the sensitized emission of SYFP2. In these experiments, the concentrations of fluorescent proteins are the same, and from the reduction of fluorescence intensity of mTurquoise2, a FRET efficiency of about $90 \%$ was estimated.

To determine the FRET efficiency at different FRET pair concentrations in C3Ms, SBFP2 was used to change the FRET pair amount in a C3M by replacing both mTurquoise 2 and SYFP2 while keeping the final protein concentration constant. Because SBFP2 has a charge distribution that is similar to those of mTurquoise2 and SYFP2 and a similar value for its PMC (0.70) and it does not absorb light at $440 \mathrm{~nm}$ (Figure 1), it has been used as an "invisible" protein substitute. ${ }^{17}$ Figure $3 \mathrm{~A}$ shows fluorescence emission spectra for C3M solutions with different FRET pair amounts consisting of $1 \mu \mathrm{M}$ total protein and $0.18 \mu \mathrm{M}$ polymer. Clearly, a FRET pair amount of $10 \%$ already shows energy transfer in C3Ms between mTurquoise 2 and SYFP2. At increasing FRET pair amounts, the donor fluorescence intensity further decreases and the acceptor fluorescence intensity increases. Related ratio $R_{\text {FRET }}$ (eq 1) increases in an almost linear fashion for increasing FRET pair amounts (Figure S1). The corresponding FRET efficiency (eq 2) follows an asymptotic curve, reaching a maximum of $\sim 0.90$ for a FRET pair amount of $100 \%$ (Figure 3B). The correlation of $R_{\text {FRET }}$ with the FRET pair content in C3Ms (Figure S1) confirms that all three proteins have similar interactions with the diblock copolymer. When the interactions of the FPs with the copolymer are significantly different, this could lead to an unbalanced uptake of the FRET pair proteins, which would be reflected in a nonlinear increase in FRET with increasing FRET pair amounts. 

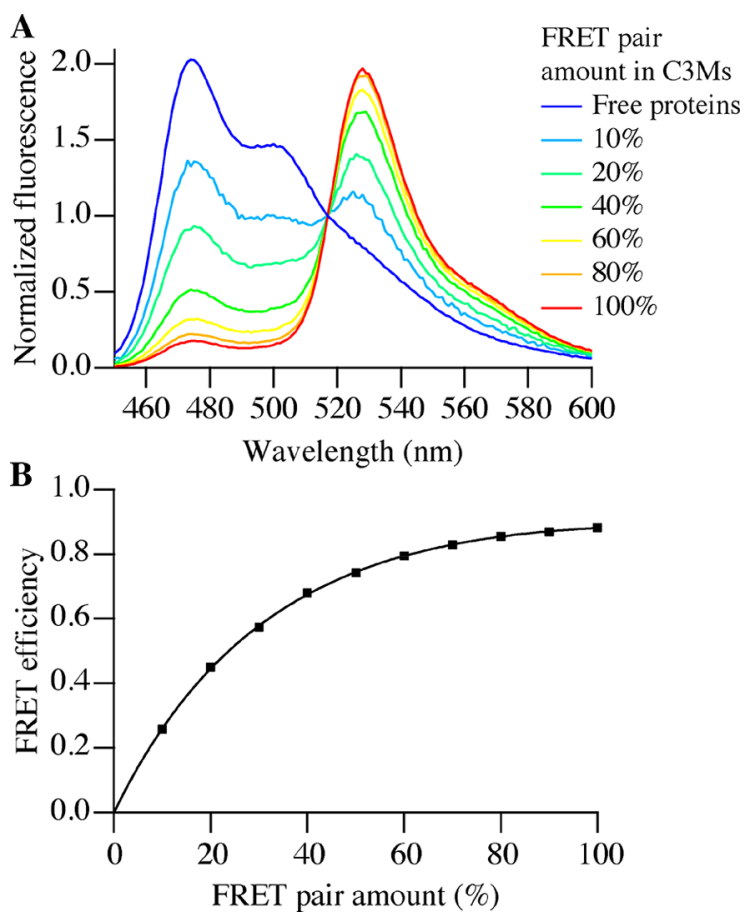

Figure 3. Normalized fluorescence intensities of the mTurquoise2 and SYFP2 FRET pair in C3Ms at different FRET pair amounts (10 to 100\%: FRET pair/SBFP2 ratio) upon donor excitation (440 nm). The total protein concentration was kept at $1 \mu \mathrm{M}$ using SBFP2 to replace both mTurquoise2 and SYFP2 to keep the FRET pair composition constant at 1:1 (mTurquoise2/SYFP2) and change the FRET pair amount in the C3Ms. The concentration of $\mathrm{P}_{2} \mathrm{MVP}_{128^{-}} b$ $\mathrm{PEO}_{477}$ was $0.18 \mu \mathrm{M}$. (A) Fluorescence emission spectra at different FRET pair amounts (normalized at $517 \mathrm{~nm}$ ). (B) FRET efficiency as a function of the FRET pair amount. The solid line is a guide to the eye.

Kinetics of Micellization. To measure the kinetics of $\mathrm{C} 3 \mathrm{M}$ formation, we monitored changes in FRET by measuring the fluorescence intensities of mTurquoise 2 and SYFP2 as a function of time. Figure 4 shows the time dependency of the ratio of SYFP2 to mTurquoise2 fluorescence intensities after mixing a solution of proteins (at a FRET pair amount of 50\%)

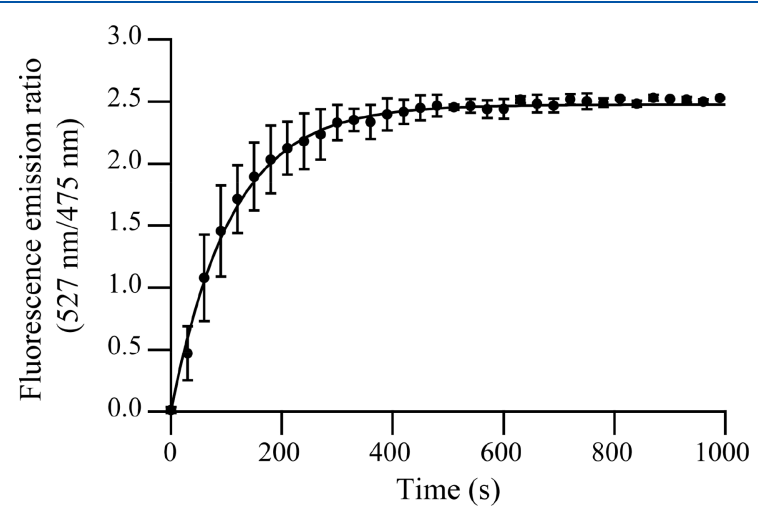

Figure 4. Fluorescence emission ratio of SYFP2 to mTurquoise2 (527 $\left.\mathrm{nm} / 475 \mathrm{~nm}, R_{\mathrm{FRET}}\right)$ as a function of time following the mixing of solutions of polymer and proteins (final concentrations: $0.25 \mu \mathrm{M}$ mTurquoise2, $0.25 \mu \mathrm{M}$ SYFP2, $0.50 \mu \mathrm{M}$ SBFP2, and $0.18 \mu \mathrm{M}$ $\left.\mathrm{P}_{2} \mathrm{MVP}_{128}-b-\mathrm{PEO}_{477}\right)$. The solid line is the curve obtained using eq 9, with $R_{\mathrm{FRET} \text {,eq }}=2.47$ and $k=9 \times 10^{-3} \mathrm{~s}^{-1}$. Error bars represent the spread of the data $\left(n_{\text {exp }}=2\right)$. with a polymer solution. The concentrations after mixing were $0.25 \mu \mathrm{M}$ mTurquoise2, $0.25 \mu \mathrm{M}$ SYFP2, $0.50 \mu \mathrm{M}$ SBFP2, and $0.18 \mu \mathrm{M} \mathrm{P}_{2} \mathrm{MVP}_{128}-b-\mathrm{PEO}_{477}$. After mixing, the amount of FRET increases, indicating the formation of C3Ms. After about $600 \mathrm{~s}$, a plateau is reached with a similar ratio of the SYFP2 to mTurquoise 2 fluorescence intensity as found for equilibrated C3Ms $(24 \mathrm{~h})$ with the same FRET pair amount $\left(R_{\text {FRET,eq }} \approx\right.$ 2.5 , Figure S1A). The time course of the concentration of mTurquoise2 not involved in FRET during C3M formation was fitted for different reaction order models (Supporting Information and Figure S2). Only the plot of the natural logarithm of the concentration of mTurquoise 2 molecules that are not involved in FRET against time $(\ln [$ mTurquoise2] vs $t)$ shows linear behavior (Figure S2B), indicating first-order kinetics. Consequently, the time-dependent change of $R_{\text {FRET }}$ was fitted to

$$
R_{\mathrm{FRET}}(t)=R_{\mathrm{FRET}, \mathrm{eq}}\left(1-\mathrm{e}^{-k t}\right)
$$

where $R_{\text {FRET,eq }}$ is the ratio of SYFP2 to mTurquoise2 fluorescence intensity at equilibrium and $k$ is the kinetic coefficient. The parameters obtained from the fit are a kinetic coefficient (first-order rate constant), $k$, of $\left(9 \times 10^{-3}\right) \pm(1 \times$ $\left.10^{-3}\right)_{\mathrm{s}^{-1}}$ and a final $R_{\mathrm{FRET} \text { eq }}$ of $2.47 \pm 0.02$ (number of experiments $\left.\left(n_{\exp }\right)=2\right)$. Thus, the relaxation time of formation of C3Ms $(\tau=1 / k)$ is about $100 \mathrm{~s}$.

Protein Exchange between Preformed C3Ms. To determine the dynamics of protein exchange between C3Ms, we monitored changes in FRET after mixing C3Ms containing SYFP2 with C3Ms containing mTurquoise 2 at different final FRET pair amounts (between 50 and 20\%, Figure 5). For

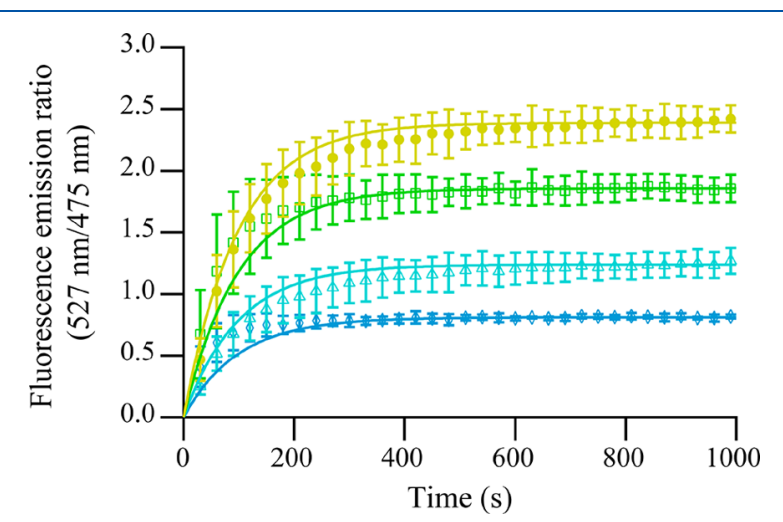

Figure 5. Fluorescence emission ratio of SYFP2 to mTurquoise2 (527 $\mathrm{nm} / 475 \mathrm{~nm}$ ) as a function of time following the exchange of proteins between SYFP2-C3Ms and mTurquoise2-C3Ms at different final FRET pair amounts: 50\% (yellow solid dots), 40\% (green open squares), 30\% (cyan open triangles), and 20\% (blue open diamonds). The total concentrations of proteins and polymer are 1 and $0.18 \mu \mathrm{M}$, respectively. The solid lines are the curves obtained using eq 9, with $k$ $=1.0 \times 10^{-2} \mathrm{~s}^{-1}$ and $R_{\mathrm{FRET}, \mathrm{eq}}=2.39$ for $50 \%, R_{\mathrm{FRET}, \mathrm{eq}}=1.86$ for $40 \%$, $R_{\mathrm{FRET} \text {,eq }}=1.24$ for $30 \%$, and $R_{\mathrm{FRET} \text { eq }}=0.81$ for $20 \%$. Error bars represent the standard deviation $\left(n_{\exp }=4\right)$.

example, for a final FRET pair amount of $20 \%$ the micelles initially contain $0.20 \mu \mathrm{M}$ SYFP2, $0.80 \mu \mathrm{M}$ SBFP2, and 0.18 $\mu \mathrm{M}$ P2MVP $128^{-b}-\mathrm{PEO}_{477}$ (for C3Ms containing SYFP2) and $0.20 \mu \mathrm{M}$ mTurquoise2, $0.80 \mu \mathrm{M}$ SBFP2, and $0.18 \mu \mathrm{M}$ $\mathrm{P}_{2} \mathrm{MVP}_{128}-b-\mathrm{PEO}_{477}$ (for C3Ms containing mTurquoise2). Accordingly, the C3Ms with the other final FRET pair amounts are mixed. 
Immediately upon mixing of the micelle solutions, the FRET amount increases, indicating the exchange of FPs between C3Ms. Within about $200 \mathrm{~s}$, a plateau is reached with ratios similar to those of the corresponding equilibrated C3Ms (0.82.3 , Figure S1A). The change in $R_{\text {FRET }}$ with time was globally fitted according to first-order reaction kinetics (eq 9, Figure S3). The obtained kinetic coefficient, $k$, is $\left(1.0 \times 10^{-2}\right) \pm(0.1$ $\left.\times 10^{-2}\right) \mathrm{s}^{-1}$ and the final ratios, $R_{\mathrm{FRET} \text {,eq }}$ are listed in Table 1 .

Table 1. Final Fluorescence Emission Ratios of SYFP2 to mTurquoise2 $\left(R_{\mathrm{FRET}, \mathrm{eq}}\right)$ after C3M Protein Exchange with a Kinetic Coefficient of $\left(1.0 \times 10^{-2}\right) \pm\left(0.1 \times 10^{-2}\right) \mathrm{s}^{-1 a}$

$\begin{array}{cc}\text { FRET pair amount }(\%) & R_{\text {FRET,eq }} \\ 20 & 0.81 \pm 0.01 \\ 30 & 1.24 \pm 0.01 \\ 40 & 1.86 \pm 0.02 \\ 50 & 2.39 \pm 0.02\end{array}$

${ }^{a}$ Standard deviations are based on multiple measurements $\left(n_{\exp }=4\right)$.

Apparently, the relaxation time for the exchange of FPs between $\mathrm{C} 3 \mathrm{Ms}$ to reach their equilibrium compositions is comparable to the relaxation time of formation, i.e., about 100 s.

Salt Stability of C3Ms. It is well known that ionic strength is a parameter that strongly affects the complexation of oppositely charged polyelectrolytes. We therefore investigated the influence of salt $(\mathrm{NaCl})$ concentration on the stability of $50 \%$ FRET pair C3Ms. Figure 6A shows the dependency of the normalized fluorescence spectra of C3Ms on ionic strength (I), considering both buffer and added salt. It can be seen that the donor fluorescence intensity increases and the acceptor fluorescence intensity decreases with increasing ionic strength
(Figure S4). The salt ions screen the electrostatic interaction between proteins and polymers, and as a result, a $50 \%$ decrease in FRET was observed at an ionic strength of $20 \mathrm{mM}$ (Figure S4). DLS experiments showed a decrease in scattering intensity above an ionic strength of $15 \mathrm{mM}$ (Figure 6C), confirming a decline in the number of C3Ms.

The stability, defined as the change in the Gibbs energy of association $\left(\Delta G_{\text {association }}^{0}\right)$ of C3Ms, was calculated over a range of ionic strengths using eq 8 , and the results are shown in Figure 6B. Extrapolation of these data to $2.5 \mathrm{mM}$ ionic strength $(0 \mathrm{mM} \mathrm{NaCl}, 10 \mathrm{mM}$ borate buffer, $\mathrm{pH} 9)$ leads to an apparent stability of $\mathrm{C} 3 \mathrm{Ms}$ of $-10.0 \mathrm{~kJ} / \mathrm{mol}$ or $-4.1 k_{\mathrm{B}} T$ (with $k_{\mathrm{B}}$ being the Boltzmann constant). We will come back to the meaning of this change in Gibbs energy in the Discussion section.

\section{DISCUSSION}

On the basis of our kinetic results, we propose a model for the formation and relaxation of FP-containing C3Ms, in line with commonly accepted two-state models for the formation of simple surfactant micelles. ${ }^{37-39}$ This model implies that immediately after the mixing of protein and diblock copolymer solutions electrically neutral protein-polymer complexes are formed. Such a process is comparable to the fast formation of large intermediate aggregates as found for PAA-containing C3Ms. ${ }^{7,9}$ Because for our systems we never observed a lightscattering intermediate, we assume the initial formation of small soluble protein-polymer complexes (SCs). Above a certain concentration, these SCs start to aggregate, forming complex coacervate microphases that grow spontaneously. While for simple, oppositely charged polyelectrolytes this growth proceeds until two macroscopic phases are formed, here growth is limited by the hydrophilic neutral chains of the
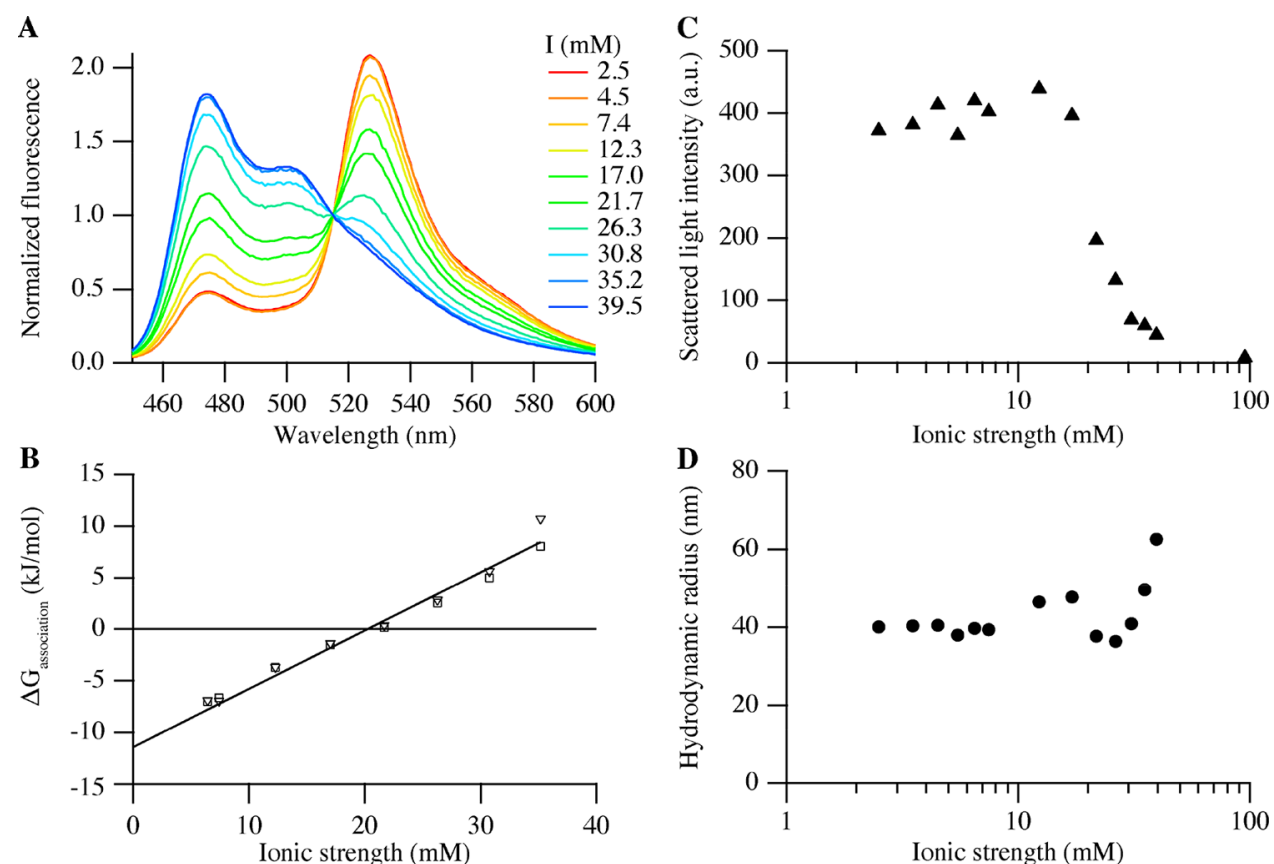

Figure 6. Salt stability of 50\% FRET pair C3Ms consisting of $0.25 \mu \mathrm{M}$ mTurquoise2, $0.25 \mu \mathrm{M}$ SYFP2, $0.50 \mu \mathrm{M}$ SBFP2, and $0.18 \mu \mathrm{M}$ P2MVP $128^{-} b$ $\mathrm{PEO}_{477}$. (A) Steady-state fluorescence emission spectra (normalized to the intensity at $517 \mathrm{~nm}$ ) at different ionic strengths $\left(n_{\exp }=3\right)$. (B) Gibbs energy of association of C3Ms as a function of ionic strength calculated from the donor (squares) and acceptor (inverted triangles) separately. (C and D) DLS results of the salt stability of C3Ms: (C) the scattered light intensity and (D) the hydrodynamic radius, both as a function of ionic strength shown on a log scale $\left(n_{\text {exp }}=1\right)$. 
diblock copolymer, which remain in the polymer-poor phase. Because growth occurs in three dimensions, the volume of the microphases increases more strongly than their surface area, hence the available area per neutral chain decreases, driving the chains together. Repulsion between these chains stops the growth of the complex coacervate cores, yielding defined sizes of the micelles. 8,37

The formation of C3Ms should have two minima in the Gibbs energy, i.e., one at low aggregation number and one at the optimum aggregation size (Figure 7). These minima

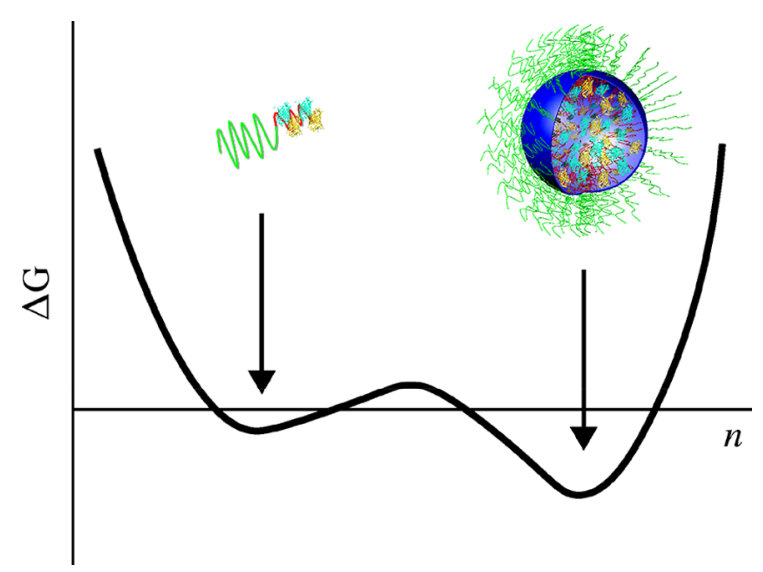

Figure 7. Schematic representation of the Gibbs energy of C3M formation as a function of aggregation number $n$. The curve shows two minima: one at low aggregation number corresponding to SCs and one at the optimum aggregation size for C3Ms.

correspond to the two states in the model: SCs and C3Ms. The maximum between these two states is the nucleation barrier for the aggregation of SCs (or the dissociation of C3Ms) and is caused by two opposite contributions: the negative "volume" contribution due to attraction between oppositely charged polyelectrolyte chains and protein molecules and a positive "surface" contribution due to the surface tension between the complex coacervate phase and the solution. Because this surface tension is quite low, ${ }^{40}$ the surface contribution is small compared to the contribution due to the aggregation of SCs, leading to a relatively low nucleation barrier.

Linking this theory to our experimental results yields a proposed model for the formation of $\mathrm{C} 3 \mathrm{Ms}$, which is summarized in Figure 8. Upon mixing of protein and diblock copolymer solutions, first a rapid formation of SCs occurs; the second step is a slower process in which C3Ms relax to their final equilibrium state due to the association and dissociation of SCs to and from the micelles.

It is reasonable to assume that the formation of SCs is diffusion-controlled. This implies that this process would be on the micro- to millisecond time scale, ${ }^{16}$ which is much too fast to be monitored by our FRET measurements. This would mean that not the formation of SCs but the increase in the volume of core material (complex coacervate microphases) is reflected by the fluorescence emission ratio of SYFP2 to mTurquoise 2 as a function of time after mixing protein and polymer solutions.

In the $\mathrm{C} 3 \mathrm{M}$ mixing experiments, changes in FRET reflect the gradual exchange of core material. Actually, this process is not that different from the relaxation process observed in the formation of C3Ms and is depicted in Figure 9: after the mixing of the two types of equilibrated C3Ms, further association and dissociation of SCs occur, allowing C3Ms to relax to their final equilibrium composition. This explains why we observed similar kinetic coefficients and relaxation times of about $100 \mathrm{~s}$ for the formation of C3Ms and for the exchange of proteins between them. An alternative mechanism for the exchange of proteins and the relaxation of micelles by "fusion and fission" is less likely ${ }^{14-16}$ because the neutral hydrophilic PEO units form a dense stabilizing corona preventing the merging of $\mathrm{C} 3 \mathrm{Ms}$.

The rate at which SCs associate with (growing) C3Ms, $r_{a}$ is proportional to the concentrations of SCs and C3Ms

$$
r_{\mathrm{a}}=k_{\mathrm{a}}[\mathrm{SC}][\mathrm{C} 3 \mathrm{M}]
$$

in which $k_{\mathrm{a}}$ is the association rate constant (Figures 8 and 9) and $[\mathrm{SC}]$ and $[\mathrm{C} 3 \mathrm{M}]$ denote molar concentrations of SCs and C3Ms, respectively. Our FRET measurements of C3M formation and the exchange between C3Ms convincingly revealed first-order kinetics; therefore, either [SC] or [C3M] should be constant on the time scale of the experiment. For that reason, eq 9 would represent pseudo-first-order kinetics. We can rule out that [SC] would be constant because the increase in FRET is due to the conversion of SCs into complex coacervate microphases (Figures 8 and 9), resulting in a decrease in [SC] during the measurements. Consequently, [C3M] should be constant to explain the order of the reaction. This is possible only if during the FRET measurements the nucleation of new micelles does not happen anymore and only micelle growth takes place.

The dissociation rate, $r_{\mathrm{d}}$ of SCs from C3Ms is proportional to the concentration of C3Ms and the average number of SCs, $n$, that the C3Ms contain

$$
r_{\mathrm{d}}=k_{\mathrm{d}} n[\mathrm{C} 3 \mathrm{M}]
$$

in which $k_{\mathrm{d}}$ is the dissociation rate constant (Figure 8). Thus, the net change in the concentration of SCs in time can be expressed as

$$
-\frac{\mathrm{d}[\mathrm{SC}]}{\mathrm{d} t}=k_{\mathrm{a}}[\mathrm{SC}][\mathrm{C} 3 \mathrm{M}]-k_{\mathrm{d}} n[\mathrm{C} 3 \mathrm{M}]=\left(k_{\mathrm{a}}[\mathrm{SC}]-k_{\mathrm{d}} n\right)[\mathrm{C} 3 \mathrm{M}]
$$
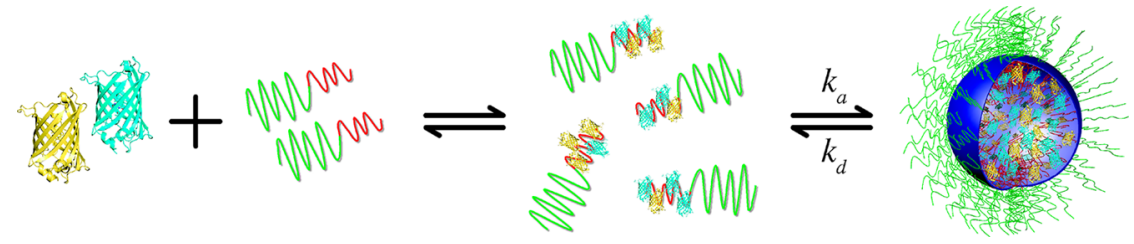

Figure 8. Schematic representation of C3M formation. Upon mixing of protein and diblock copolymer solutions, a rapid formation of SCs occurs. These SCs are assumed to be near-neutral protein-polymer complexes. Subsequently, the formation of C3Ms is a slower process in which SCs continuously associate to and dissociate from C3Ms, with the corresponding rate constants $k_{\mathrm{a}}$ and $k_{\mathrm{d}}$, respectively. 


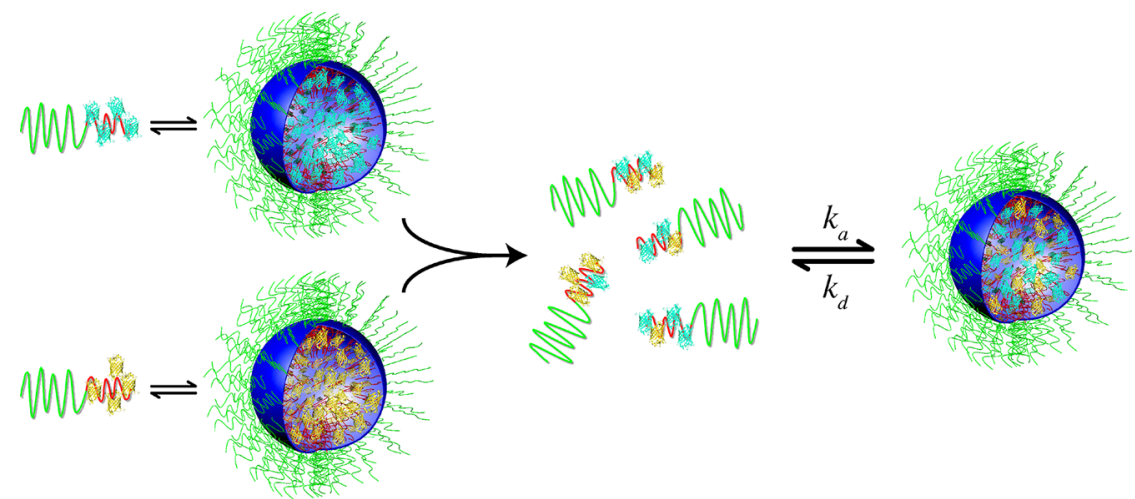

Figure 9. Schematic representation of protein exchange between C3Ms. Upon mixing of equilibrated C3Ms with their corresponding SCs, the SCs become mixed. These mixed SCs associate and dissociate with the C3Ms with rate constants of $k_{\mathrm{a}}$ and $k_{\mathrm{d}}$, respectively, resulting in a population of mixed C3Ms.

At equilibrium, $-\mathrm{d}[\mathrm{SC}] / \mathrm{d} t$ is zero, $n$ is constant, and eq 12 reduces to

$$
[\mathrm{SC}]_{\mathrm{eq}}=k_{\mathrm{d}} n / k_{\mathrm{a}}
$$

This implies that in the final state and provided that the concentrations of protein and polymer are high enough to form micelles, the concentration of SCs is independent of the total concentration of protein and polymer. The concentration of SCs at equilibrium approximately corresponds to the critical micelle concentration (CMC), which we have expressed previously in terms of the free protein concentration. ${ }^{1}$ For large aggregation numbers, $n$, the CMC is related to the Gibbs energy of micellization: ${ }^{41}$

$$
\Delta G_{\text {micellization }}=R T \ln (\mathrm{CMC})
$$

For simplicity, we assume that soluble complexes correspond to one polymer chain (with the number of charged groups being about +112 ) with on average 10 to 11 protein molecules (with a net charge of about -10 ), resulting in more or less electrically neutral moieties. Previously, the CMC for encapsulated EGFP was found at $100 \mathrm{nM}$ protein at a PMC of $0.65 .^{1}$ As explained in the Introduction, the CMCs for the FPs used in the present study are very similar. Expressing the CMC via the diblock copolymer concentration, a value of 18 $\mathrm{nM}$ is found. By substituting this value into eq 14 , we estimate the Gibbs energy of micellization to be $-44 \mathrm{~kJ} / \mathrm{mol}$ or $-18 k_{\mathrm{B}} T$ at an ionic strength of $2.5 \mathrm{mM}$. This is the free-energy gain when an SC associates with a C3M.

The decrease in FRET during the salt titration reflects an increase in the average distance between mTurquoise 2 and SYFP2. This is probably due to the disintegration of the micellar cores because salt ions screen the attractive electrostatic interaction between the protein molecules and the charged block of the polymer, thereby lowering the entropic contribution of counterion release to the stability. We assume that electrostatic interactions play a role, particularly in the formation of SCs, because the formation of C3Ms results from the assembly of these near-neutral entities. Therefore, the addition of salt would especially affect the stability of SCs. The Gibbs energy of association obtained from the salt titration reflects this stability. At an ionic strength of $2.5 \mathrm{mM}$, $\Delta G_{\text {association }}$ is $-10.0 \mathrm{~kJ} / \mathrm{mol}$ or $-4.1 k_{\mathrm{B}} T$ (Figure $6 \mathrm{~B}$ ). Together with the Gibbs energy of the insertion of an SC into the micelle, $\Delta G_{\text {micellization }}$ (eq 14), a rough estimate of the overall Gibbs energy of formation of a C3M, is

$$
\Delta G_{\mathrm{C} 3 \mathrm{M}}=n\left(\Delta G_{\text {association }}+\Delta G_{\text {micellization }}\right)
$$

with $n$ being the aggregation number of SCs in a C3M. From the number of charges on the polymer (112) and the protein (9 to 11), we assume that on average 10 to 11 protein molecules are associated with 1 polymer chain. About 450 proteins are encapsulated in $1 \mathrm{C} 3 \mathrm{M}^{1}$ therefore, on the basis of charge, about 40 diblock copolymers together with the proteins make up $1 \mathrm{C} 3 \mathrm{M}$. Taking the aggregation number equal to the number of diblock copolymers per C3M, i.e., $n \approx$ 40 , eq 15 yields a value for $\Delta G_{\mathrm{C} 3 \mathrm{M}}$ of about $-2160 \mathrm{~kJ} / \mathrm{mol}$ or $-880 k_{B} T /$ micelle (at $2.5 \mathrm{mM}$ ionic strength).

Although the overall stability of C3Ms thus seems to be quite high, their structure is highly dynamic and SCs with proteins are easily dissociated from and reassociated with $\mathrm{C} 3 \mathrm{Ms}$, resulting in a rapid exchange of proteins between the micelle core and bulk solution. This has a huge impact on the practical applicability of $\mathrm{C} 3 \mathrm{Ms}$ as protein carriers: the protein molecules reside part of the time outside the C3Ms and thus are unprotected. In addition, half of the micelles dissociate at an ionic strength of $20 \mathrm{mM}$, which makes these systems inapplicable under physiological conditions.

To improve the stability and reduce the dynamics of C3Ms, several options exist. For example, one could choose to add an additional homopolymer to obtain three-component $\mathrm{C} 3 \mathrm{Ms}$; as shown by Lindhoud et al., ${ }^{4}$ such C3Ms can have very long relaxation times. The cause of this phenomenon is not clear, however, and a disadvantage is that much less protein molecules per micelle can be accommodated. Alternatively, one could modify the protein, as has been done by Obermeyer et al., ${ }^{42}$ who increased the net charge of several proteins by converting positively charged lysine residues to negatively charged residues. These negatively charged proteins were encapsulated with strong polycations, resulting in the improved stability of the micelles to increased ionic strength as the net charge on the protein increased. In view of our findings, a very effective option for trapping proteins would be to cross-link the charged blocks of the diblock copolymers in the $\mathrm{C} 3 \mathrm{M}$ cores by using amide bonds. ${ }^{43,44}$ By doing so, it is not possible for proteins to leave micelles in the form of almost neutral SCs, and the energy barrier to escape on their own will be much higher. An additional advantage of this approach is that the proteins do not need to be modified. 


\section{CONCLUSIONS}

In this article, the formation of protein-containing C3Ms, the protein exchange between them, and their salt stability have been studied using FRET. Energy transfer was followed between mTurquoise2 and SYFP2, which come in close proximity to each other in $\mathrm{C} 3 \mathrm{Ms}$ upon mixing with diblock copolymer $\mathrm{P}_{2} \mathrm{MVP}_{128}-b-\mathrm{PEO}_{477}$. At an ionic strength of about $20 \mathrm{mM}$, FRET in such a micellar solution reduces to $50 \%$ of its initial value as a result of the weakening of the electrostatic interactions between polymers and proteins. Both the formation of $\mathrm{C} 3 \mathrm{Ms}$ and the protein exchange between $\mathrm{C} 3 \mathrm{Ms}$ follow first-order kinetics with a relaxation time of about $100 \mathrm{~s}$, implying that the structures are dynamic. The similarity between both processes can be explained by a two-state model in which the equilibration of C3Ms to their final size and composition involves the association and dissociation of small near-neutral protein-polymer complexes (SCs). On the basis of the salt stability results, the proposed model, and the assumption that an SC comprises 1 diblock copolymer molecule and about 10 protein molecules, we estimate the overall Gibbs energy of $\mathrm{C} 3 \mathrm{M}$ formation to be $-880 k_{B} T /$ micelle.

\section{ASSOCIATED CONTENT}

\section{S Supporting Information}

The Supporting Information is available free of charge on the ACS Publications website at DOI: 10.1021/acs.langmuir.8b01272.

Fluorescence emission ratio of SYFP2 to mTurquoise2 in C3Ms, C3M formation and protein exchange kinetics, and normalized donor and acceptor fluorescence intensities as a function of ionic strength (PDF)

\section{AUTHOR INFORMATION}

\section{Corresponding Author}

*E-mail: janwillem.borst@wur.nl. Tel: +31 317483724.

ORCID $\odot$

Willem J. H. van Berkel: 0000-0002-6551-2782

Jan Willem Borst: 0000-0001-8176-9302

\section{Author Contributions}

A.N. purified SBFP2, mTurquoise2, and SFYP2, analyzed data, and wrote the manuscript. E.H. and J.W.B. performed the experiments. A.H.W. assisted with data interpretation. A.N., E.H., J.M.K., W.J.H.v.B., and J.W.B. designed the experiments. A.H.W., J.M.K., W.J.H.v.B., and J.W.B. contributed to the writing of the manuscript.

\section{Notes}

The authors declare no competing financial interest.

\section{ACKNOWLEDGMENTS}

Financial support from the Graduate School VLAG (Wageningen, The Netherlands) is gratefully acknowledged. We thank Dr. Joachim Goedhart (University of Amsterdam) for providing us with the pRSETb vector containing SBFP2 and Prof. Frans Leermakers (Wageningen University and Research) for fruitful discussions.

\section{REFERENCES}

(1) Nolles, A.; Westphal, A. H.; de Hoop, J. A.; Fokkink, R. G.; Kleijn, J. M.; van Berkel, W. J. H.; Borst, J. W. Encapsulation of GFP in complex coacervate core micelles. Biomacromolecules 2015, 16 (5), $1542-1549$.

(2) Nolles, A.; van Dongen, N. J. E.; Westphal, A. H.; Visser, A. J. W. G.; Kleijn, J. M.; van Berkel, W. J. H.; Borst, J. W. Encapsulation into complex coacervate core micelles promotes EGFP dimerization. Phys. Chem. Chem. Phys. 2017, 19 (18), 11380-11389.

(3) Lindhoud, S.; de Vries, R.; Norde, W.; Cohen Stuart, M. A. Structure and stability of complex coacervate core micelles with lysozyme. Biomacromolecules 2007, 8 (7), 2219-2227.

(4) Lindhoud, S.; Norde, W.; Cohen Stuart, M. A. Reversibility and relaxation behavior of polyelectrolyte complex micelle formation. J. Phys. Chem. B 2009, 113 (16), 5431-5439.

(5) Harada, A.; Kataoka, K. Pronounced activity of enzymes through the incorporation into the core of polyion complex micelles made from charged block copolymers. J. Controlled Release 2001, 72 (1-3), $85-91$.

(6) Yuan, X. F.; Yamasaki, Y.; Harada, A.; Kataoka, K. Characterization of stable lysozyme-entrapped polyion complex (PIC) micelles with crosslinked core by glutaraldehyde. Polymer 2005, 46 (18), $7749-7758$.

(7) Cohen Stuart, M. A.; Besseling, N. A. M.; Fokkink, R. G. Formation of micelles with complex coacervate cores. Langmuir 1998, 14 (24), 6846-6849.

(8) Voets, I. K.; de Keizer, A.; Cohen Stuart, M. A. Complex coacervate core micelles. Adv. Colloid Interface Sci. 2009, 147-148, $300-318$.

(9) Hofs, B.; Voets, I. K.; de Keizer, A.; Cohen Stuart, M. A. Comparison of complex coacervate core micelles from two diblock copolymers or a single diblock copolymer with a polyelectrolyte. Phys. Chem. Chem. Phys. 2006, 8 (36), 4242-4251.

(10) de Vries, R.; Cohen Stuart, M. A. Theory and simulations of macroion complexation. Curr. Opin. Colloid Interface Sci. 2006, 11 (5), 295-301.

(11) Kriz, J.; Dybal, J.; Dautzenberg, H. Cooperative interactions of unlike macromolecules: 3. NMR and theoretical study of the electrostatic coupling of sodium polyphosphates with diallyl(dimethyl)ammonium chloride-acrylamide copolymers. J. Phys. Chem. A 2001, 105 (31), 7486-7493.

(12) Besseling, N. A. M.; Cohen Stuart, M. A. Self-consistent field theory for the nucleation of micelles. J. Chem. Phys. 1999, 110 (11), $5432-5436$

(13) Hofs, B.; de Keizer, A.; Cohen Stuart, M. A. On the stability of (highly aggregated) polyelectrolyte complexes containing a chargedblock-neutral diblock copolymer. J. Phys. Chem. B 2007, 111 (20), 5621-5627.

(14) Holappa, S.; Kantonen, L.; Andersson, T.; Winnik, F.; Tenhu, $\mathrm{H}$. Overcharging of polyelectrolyte complexes by the guest polyelectrolyte studied by fluorescence spectroscopy. Langmuir 2005, 21 (24), 11431-11438.

(15) Dormidontova, E. E. Micellization kinetics in block copolymer solutions: Scaling model. Macromolecules 1999, 32 (22), 7630-7644.

(16) Zhang, J.; Chen, S.; Zhu, Z.; Liu, S. Stopped-flow kinetic studies of the formation and disintegration of polyion complex micelles in aqueous solution. Phys. Chem. Chem. Phys. 2014, 16 (1), $117-127$.

(17) Nolles, A.; Westphal, A. H.; Kleijn, J. M.; van Berkel, W. J. H.; Borst, J. W. Colorful packages: Encapsulation of fluorescent proteins in complex coacervate core micelles. Int. J. Mol. Sci. 2017, 18 (7), 1557.

(18) Goedhart, J.; von Stetten, D.; Noirclerc-Savoye, M.; Lelimousin, M.; Joosen, L.; Hink, M. A.; van Weeren, L.; Gadella, T. W. J.; Royant, A. Structure-guided evolution of cyan fluorescent proteins towards a quantum yield of $93 \%$. Nat. Commun. 2012, 3, 751 .

(19) Kremers, G. J.; Goedhart, J.; van Munster, E. B.; Gadella, T. W. J. Cyan and yellow super fluorescent proteins with improved brightness, protein folding, and FRET Förster radius. Biochemistry 2006, 45 (21), 6570-6580.

(20) Chong, S.; Mersha, F. B.; Comb, D. G.; Scott, M. E.; Landry, D.; Vence, L. M.; Perler, F. B.; Benner, J.; Kucera, R. B.; Hirvonen, C. 
A.; Pelletier, J. J.; Paulus, H.; Xu, M. Q. Single-column purification of free recombinant proteins using a self-cleavable affinity tag derived from a protein splicing element. Gene 1997, 192 (2), 271-281.

(21) Evans, T. C., Jr.; Xu, M. Q. Intein-mediated protein ligation: harnessing nature's escape artists. Biopolymers 1999, 51 (5), 333-342.

(22) Xu, M. Q.; Paulus, H.; Chong, S. Fusions to self-splicing inteins for protein purification. Methods Enzymol. 2000, 326, 376-418.

(23) Kremers, G. J.; Goedhart, J.; van den Heuvel, D. J.; Gerritsen, H. C.; Gadella, T. W. J. Improved green and blue fluorescent proteins for expression in bacteria and mammalian cells. Biochemistry 2007, 46 (12), 3775-3783.

(24) Rostkowski, M.; Olsson, M. H.; Sondergaard, C. R.; Jensen, J. $\mathrm{H}$. Graphical analysis of $\mathrm{pH}$-dependent properties of proteins predicted using PROPKA. BMC Struct. Biol. 2011, 11, 6.

(25) Olsson, M. H. M.; Sondergaard, C. R.; Rostkowski, M.; Jensen, J. H. PROPKA3: Consistent Treatment of Internal and Surface Residues in Empirical pK(a) Predictions. J. Chem. Theory Comput. 2011, 7 (2), 525-537.

(26) Provencher, S. W. Contin - a general-purpose constrained regularization program for inverting noisy linear algebraic and integral-equations. Comput. Phys. Commun. 1982, 27 (3), 229-242.

(27) Provencher, S. W. A constrained regularization method for inverting data represented by linear algebraic or integral equations. Comput. Phys. Commun. 1982, 27 (3), 213-227.

(28) Förster, T. Zwischenmolekulare energiewanderung und fluoreszenz. Ann. Phys. 1948, 437, 55-75.

(29) Stryer, L. Fluorescence energy transfer as a spectroscopic ruler. Annu. Rev. Biochem. 1978, 47, 819-846.

(30) Zhang, J.; Campbell, R. E.; Ting, A. Y.; Tsien, R. Y. Creating new fluorescent probes for cell biology. Nat. Rev. Mol. Cell Biol. 2002, 3 (12), 906-918.

(31) Piston, D. W.; Kremers, G. J. Fluorescent protein FRET: the good, the bad and the ugly. Trends Biochem. Sci. 2007, 32 (9), 407414.

(32) Hohlbein, J.; Craggs, T. D.; Cordes, T. Alternating-laser excitation: single-molecule FRET and beyond. Chem. Soc. Rev. 2014, 43 (4), 1156-1171.

(33) Steinberg, I. Z. Nonradiative energy transfer in systems in which rotatory brownian motion is frozen. J. Chem. Phys. 1968, 48 (6), 2411-2413.

(34) Eisinger, J.; Dale, R. E. Interpretation of intramolecular energy transfer experiments. J. Mol. Biol. 1974, 84 (4), 643-647.

(35) Ormö, M.; Cubitt, A. B.; Kallio, K.; Gross, L. A.; Tsien, R. Y.; Remington, S. J. Crystal structure of the Aequorea victoria green fluorescent protein. Science 1996, 273 (5280), 1392-1395.

(36) Yang, F.; Moss, L. G.; Phillips, G. N. J. The molecular structure of green fluorescent protein. Nat. Biotechnol. 1996, 14 (10), 12461251.

(37) Sams, P. J.; Wyn-Jones, E.; Rassing, J. A new model describing the kinetics of micelle formation from chemical relaxation studies. Chem. Phys. Lett. 1972, 13 (3), 233-236.

(38) Laughlin, R. G. The Aqueous Phase Behavior of Surfactants; Academic Press: London, 1994.

(39) Leermakers, F. A. M.; Eriksson, J. C.; Lyklema, J. Association Colloids and Their Equilibrium Modelling. In Fundamentals of Interface and Colloid Science: Soft Colloids; Lyklema, J., Ed.; Academic Press: Amsterdam, 2005; Vol. 5.

(40) Spruijt, E.; Sprakel, J.; Cohen Stuart, M. A.; van der Gucht, J. Interfacial tension between a complex coacervate phase and its coexisting aqueous phase. Soft Matter 2010, 6 (1), 172-178.

(41) Phillips, J. N. The energetics of micelle formation. Trans. Faraday Soc. 1955, 51 (4), 561-569.

(42) Obermeyer, A. C.; Mills, C. E.; Dong, X. H.; Flores, R. J.; Olsen, B. D. Complex coacervation of supercharged proteins with polyelectrolytes. Soft Matter 2016, 12 (15), 3570-3581.

(43) Bourouina, N.; Cohen Stuart, M. A.; Kleijn, J. M. Complex coacervate core micelles as diffusional nanoprobes. Soft Matter 2014, 10 (2), 320-331.
(44) Kim, J. O.; Ramasamy, T.; Yong, C. S.; Nukolova, N. V.; Bronich, T. K.; Kabanov, A. V. Cross-linked polymeric micelles based on block ionomer complexes. Mendeleev Commun. 2013, 23 (4), 179186 